\author{
Military Technical College \\ Kobry El-Kobba \\ Cairo, Egypt
}

12-th International Conference

on

Aerospace Sciences \&

Aviation Technology

\title{
Performance Study of Convolutional Coded OFDM
}

\author{
A. A. Elrahman, M. M. Salah, Y. Z. Mohasseb*, and M. M. Mokhtar
}

\begin{abstract}
Orthogonal Frequency Division Multiplexing (OFDM) is a widely known modulation scheme used for high speed data transmission (like video) on multipath fading channels. The multipath components of these channels cause a frequency selective fading that seriously degrade the OFDM transmission, due to the erroneous exist on the received data. Time guard-interval is used to reduce intersymbol interference (ISI) and Intercarrier interference (ICI). Convolutional coding protects digital data from errors by selectively introducing redundancies in the transmitted data.

In this paper a study of a combined use of Convolutional coding and OFDM technique is presented. The effect of coding rates and constraint lengths of the Convolutional code and the time guard interval of the OFDM symbols are studied under frequency selective environment through computer simulation. Recommendations for design issues are also discussed.
\end{abstract}

KEYWORDS: OFDM, Convolutional coding.

* Egyptian Armed Forces 


\section{INTRODUCTION}

With the rapid growth of digital wireless (video, voice and data) communications in recent years, the need of high speed mobile data transmission has increased. The channel impulse response can extend over many symbol periods at high bit rates, which leads to intersymbol interference (ISI). Orthogonal frequency division multiplexing (OFDM) has been found to be one of the most effective techniques in combating ISI [1, 2].

The OFDM is a bandwidth efficient multicarrier transmission scheme for digital communications that was first proposed by Chang in 1966 [3]. It has gained a lot of popularity among the broadband community in the last few years due to its high spectral efficiency, and multipath delay spread tolerance. The main idea of the OFDM is to split a high-rate data stream into a number of lower rate streams that are transmitted simultaneously over a number of spectrally overlapping orthogonal subcarriers, which can be accomplished perfectly utilizing the Discrete Fourier Transform (DFT) as first described by Darlington in 1970 [4]. With the recent evolution of digital signal processing (DSP) chips, OFDM has become practical to implement. So the modulation of these sub-carriers can be represented as Inverse Fast Fourier Transform (IFFT). Both the ISI and the ICI can be completely eliminated using OFDM by adding time guard interval $(\mathrm{Gl})$ between symbols. The length of the $\mathrm{Gl}$ is made equal to or greater than the time spread of the channel $[1,5]$.

The applications that use OFDM technology have been increased over the last few years, including the European Digital Audio Broadcasting (DAB), Digital Video Broadcasting (DVB), standardized by the European Telecommunication Institute (ETSI) in 1995, and 1997 respectively, wireless local area networks (WLANs) (802.11a) in 1998 [6], and Japanese integrated services digital broadcasting (ISDB) [7]. Using guard interval is limited by bandwidth efficiency. In this paper we consider the benefits of using a convolutional coding strategy in conjunction with OFDM modulation for different parameters values, such as coding rate and constraint length of the convolutional coding, and the time guard interval of the OFDM signaling to achieve the desired performance.

This paper is organized as follow. Section II presents a brief overview for OFDM signaling and convolutional coding. Section III includes the description of the system model used and system model validation. Section IV Simulation results are presented. Section VI summarizes the paper.

\section{BACKGROUND OVERVIEW}

\section{1 Orthogonal Frequency Division Multiplexing (OFDM)}

OFDM uses the Inverse Fast Fourier Transform (IFFT) to modulate a serially transmitted signal into parallel orthogonal signals. At the receiver, the demodulation is achieved by the Fast Fourier transform (FFT). Due to the low complexity of the IFFT and the FFT, OFDM is an attractive modulation scheme for many wireless and wireline communications applications.

OFDM transmission scheme consists of modulator, serial to parallel converter, IFFT, guard interval insertion and parallel to serial converter. 
The receiver consists of serial to parallel converter, guard interval removal, FFT, parallel to serial converter and demodulator. The scheme of an OFDM system is shown in Fig. 1

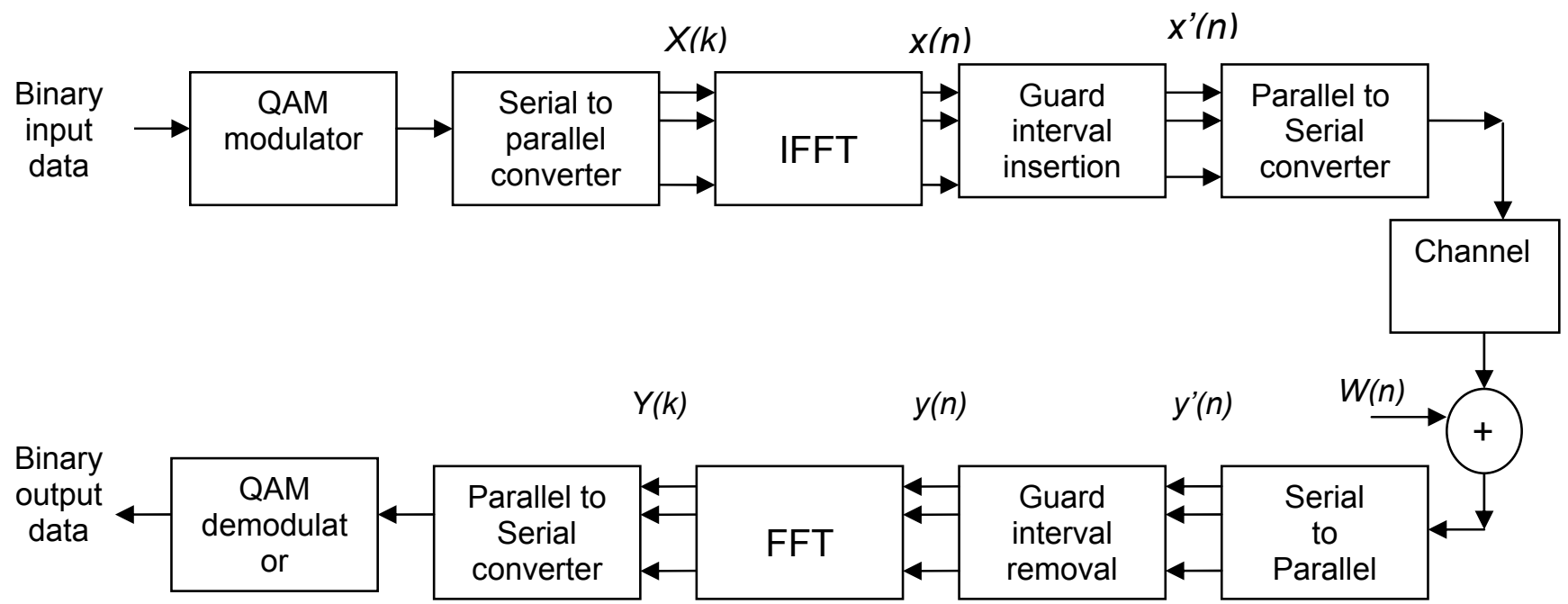

Fig.1. A Transmission and reception scheme based on OFDM.

The binary information is first grouped and mapped according to the modulation used, then converted to a parallel form. The IFFT block is used to transform the data sequence $X(k)$ into time domain sequence $x(n)$ expressed as [8]:

$$
\begin{aligned}
x(n) & =\operatorname{IFFT}\{X(k)\} \\
& =\sum_{k=0}^{N-1} X(k) e^{j(2 \pi k n / N)} \quad n=0,1,2 \ldots N-1
\end{aligned}
$$

where $N$ is the FFT length. The length of the guard interval is chosen to be larger than the expected delay spread to prevent the intersymbol interference (ISI). This guard time includes the cyclically extended part of the OFDM symbol in order to eliminate Intercarrier interference (ICl).

The received signal through a frequency selective time varying fading channel with additive noise is given by:

$$
y^{\prime}(n)=x^{\prime}(n) \otimes h(n)+w(n)
$$

where $w(n)$ is the Additive White Gaussian noise (AWGN) and $h(n)$ is the channel impulse response, and the prime denotes a cyclically extended signal.

At the receiver, the guard interval is removed giving $y(n)$, and a reverse process is performed through a FFT block to yield [8]:

$$
\begin{aligned}
Y(k) & =F F T\{y(n)\} \\
& =\frac{1}{N} \sum_{n=0}^{N-1} y(n) e^{-j(2 \pi k n / N)} \quad k=0,1,2 \ldots, N-1
\end{aligned}
$$




\section{II.2 Convolutional Codes}

Channel coding protects digital data from errors by selectively introducing redundancies in the transmitted data. Coding involves adding extra bits to the data stream so that the decoder can reduce or correct errors at the output of the receiver. Channel coding techniques can be divided into three categories; block codes, convolutional codes, and concatenated codes.

Convolutional codes are powerful coding schemes for wireless mobile communication systems. It can achieve a larger coding gain than a block coding with the same complexity. A convolutional code is generated by passing the information sequence to be transmitted through a linear finite state shift register. The convolutional code rate is defined as $r=k / n$, where $n$ is the number of coded symbols produced when $k$ information bits are shifted to the convolutional encoder. The parameter $K$ is called the constraint length of the convolutional code [9].

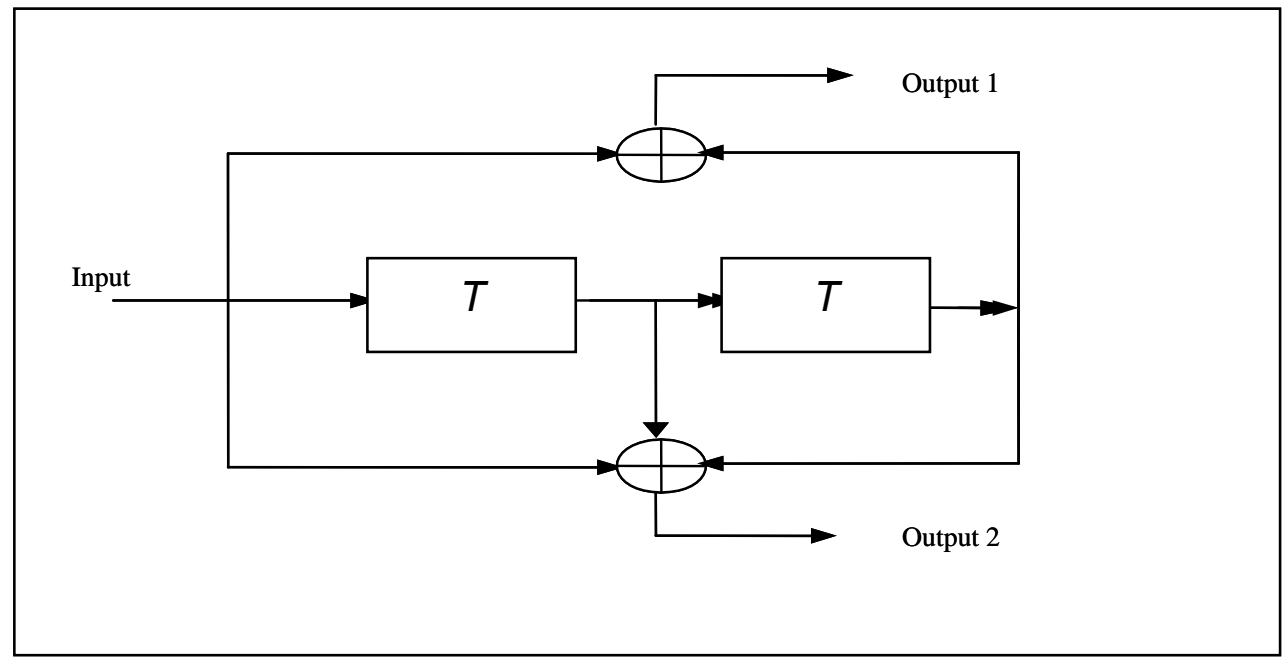

Fig. 2. Configuration of the convolutional encoder with $1 / 2$ code rate and $K=.3$.

Fig. 2 shows the configuration of the convolutional encoder with a coding rate of $1 / 2$ with constraint length $(K)$ of 3 . The convolutional encoder consists of $(K-1=2)$ shift registers and two modulo- 2 adders connected to some of the shift registers. The input data sequence is fed to the shift registers, and two output data are calculated using the contents of the shift registers. Two coded bits are produced when a single source bit is input. The generator polynomials determine the encoding process, and they are more conveniently expressed in the octal form. Let us consider the binary convolutional encoder shown in Fig. 2, the first function generator $g_{1}$, the upper one, is [101], and the second function generator $g_{2}$, the lower one, is [111], which are represented in the octal form by 5 and 7 respectively. The list of best known generator codes, based on free distance criteria, of rate $1 / 2$ for $K=3$ to 9 , and rate $1 / 3$ for $K=3$ to 8 , was reported in [9]. There are a number of techniques for decoding convolutional codes [9]; Viterbi decoding algorithm, Fano's sequential decoding, the stack algorithm, and feedback decoding. The most important of these methods is the Viterbi algorithm which performs maximum likelihood decoding of convolutional codes. 


\section{SYSTEM MODEL}

Fig. 3 illustrates the proposed system under study. As shown, Convolutional coding is integrated into the OFDM system to enhance the performance improvements in multipath fading channels. The binary input information is first encoded using convolution coding at code rate $r$, and then OFDM modulation at transmission. On the receiver side, the OFDM signal is demodulated and decoded.

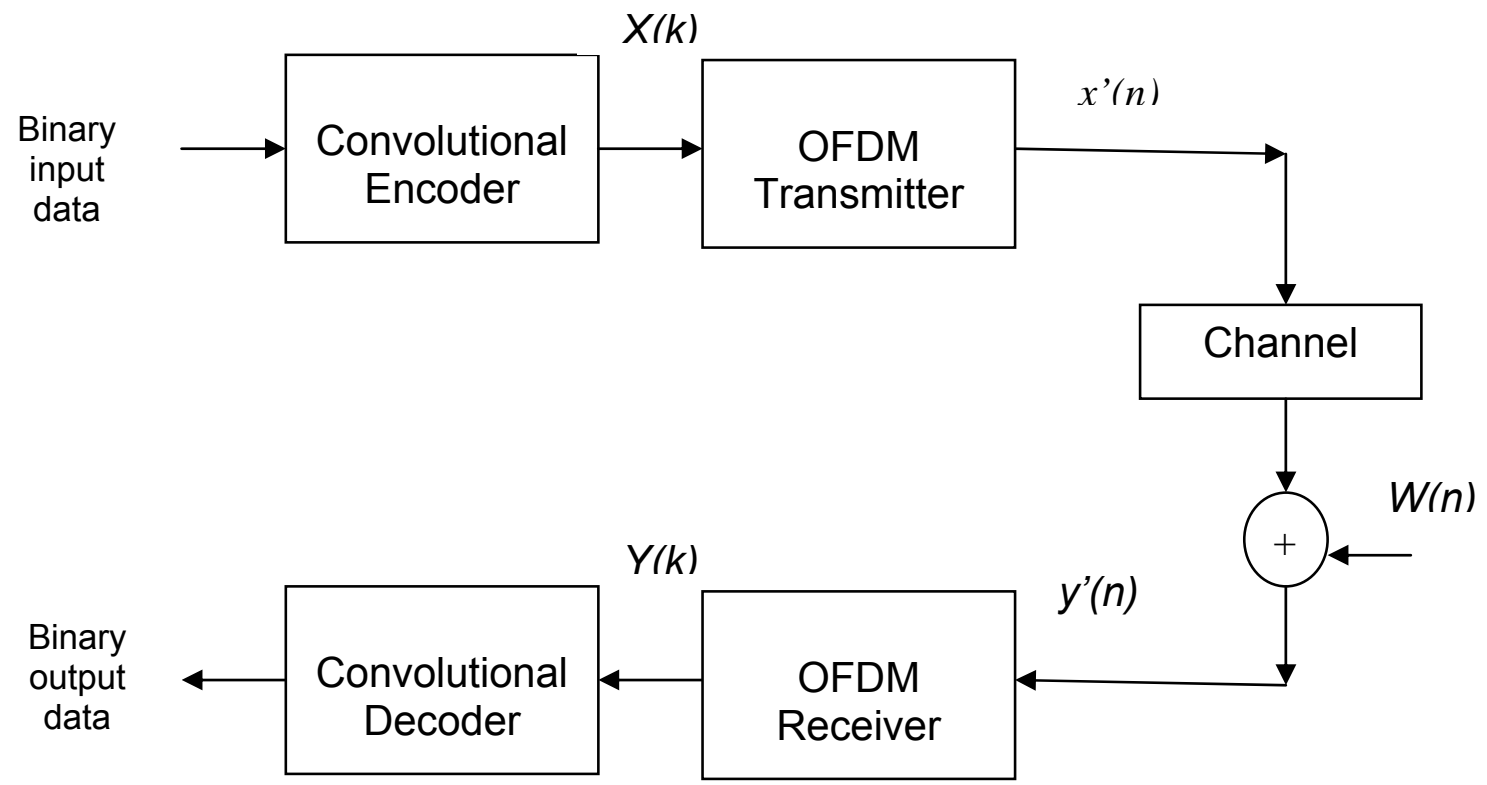

Fig. 3. The OFDM proposed system under study

The transmitted signal $x^{\prime}(n)$ will pass through the frequency selective time varying fading channel with additive noise. In this study we apply two path faded channel model. The channel impulse response can be represented as [10]:

$$
\left.h(t)=\frac{1}{\sqrt{L}} \sum_{m=1}^{L} e^{j\left(\theta_{m}+2 \pi\right.} f_{D m^{t}}\right) \delta\left(t-T_{m}\right)
$$

Where $L$ is the number of reflected multipaths, $I_{m}$ is the delay, $\theta_{m}$ is the phase rotation and $f_{D m}$ is the Doppler frequency offset of the $m^{\text {th }}$ path. At the receiver, the output of the $k^{\text {th }}$ sub-channel for the $n^{\text {th }}$ block can be written as two terms:

$$
Y_{n, k}=X_{n, k}+I_{n, k}
$$

Where $X_{n, k}$ represents the direct path term, while $I_{n, k}$ represents the interfering term due to the echo of the preceding blocks. If the guard-interval time is less than the maximum delay spread on the channel, the orthogonality conditions do not hold any more. The interfering term will be: 


$$
I_{n, k}=A e^{j\left(\theta+2 \pi f_{D^{n}} T^{\prime}{ }^{\prime}\right)}\left[\sum_{l=0}^{N-1} X_{n-1, l} \Lambda_{l, k}(\tau)+\sum_{l=0}^{N-1} X_{n, l} \mu_{l, k}(\tau)\right]
$$

Where $X_{n, l}$ is the data sequence being transmitted on the $I^{\text {th }}$ sub-channel in the $n^{\text {th }}$ block, $A$ is the attenuation, $f_{D}$ is the Doppler frequency, $T_{b}$ is the effective OFDM block duration (i.e. $T_{b}=N T_{s}$, where $T_{s}$ is the period of each symbol). The addition of a guard interval $T_{g}$, gives a new total block duration $T_{b}$ expressed as:

$T^{\prime}{ }_{b}=T_{b}+T_{g}$

$\lambda_{l, k}$ and $\mu_{l, k}$ determine the level of dependence between the sub-channel fading effects on the $N$-Carriers, which have been found to be given as [10]:

$\lambda_{I, k}(T)= \begin{cases}\left(\frac{T-T_{g}}{T_{b}}\right) e^{j 2 \pi k\left(T_{b^{-}-T}\right) / T_{b}} & \text { for } \quad l=k \\ e^{j \pi\left[2 I\left(T{ }^{\prime}{ }^{-}-T\right) / T_{b}+(I-k)\left(T-T_{g}\right)\right] \sin \left[\pi(I-k)\left(T-T_{g}\right) / T_{b}\right]} & \text { otherwise }\end{cases}$

$\mu_{I, k}(T)= \begin{cases}\left(\frac{T_{b}-T+T_{g}}{T_{b}}\right) e^{j 2 \pi k(T T) / T_{b}} & \text { for } \quad l=k \\ e^{-j \pi\left[-2 / T / T_{b}+(I-k)\left(T-T_{g}\right) / T_{b}\right]} \frac{\sin \left[\pi(I-k)\left(T-T_{g}\right) / T_{b}\right]}{\pi(I-k)} & \text { otherwise }\end{cases}$

For the multipath channel model considered here, the received signal is:

$Y_{n, k}=\frac{\mu_{k, k}}{\sqrt{L}} X_{n, k}+\sum_{I=0, I \neq k}^{N-1} \frac{\mu_{l, k}}{\sqrt{L}} X_{n, I}+\sum_{I=0, I \neq k}^{N-1} \frac{\lambda_{I, k}}{\sqrt{L}} X_{n-1, I} \quad$ for $\quad k=0,1,2 \ldots, N-1$

where, where $X_{n, k}$ is the data sequence being transmitted on the $k^{\text {th }}$ sub-channel in the $n^{\text {th }}$ block, and

$$
\begin{aligned}
& \Lambda_{l, k}=\sum_{\tau \in\left(\tau T_{m}>T_{g}\right)} H_{m} \Lambda_{l, k}(T) \\
& \mu_{l, k}=\sum_{\tau \in\left(\tau_{m}>T_{g}\right)} H_{m} \mu_{l, k}(\tau) \\
& \mu_{k, k}=\sum_{\tau \in\left(\tau_{\tau_{m}} \leq T_{g}\right)} H_{m} e^{j 2 \pi} f_{k}^{\tau}+\sum_{\tau \in\left(\tau_{m}>T_{g}\right)} H_{m} \mu_{k, k}(\tau)
\end{aligned}
$$


and the channel coefficients for each reflected path $H_{m}$ are given by:

$$
H_{m}=e^{j\left(\theta_{m}+2 \pi f_{D m}^{n} T^{\prime}{ }_{b}\right)}
$$

and each sub-carrier frequency $f_{k}$ is given by:

$$
f_{k}=f_{c}+k / T_{b} \quad \text { for } k=0,1,2 \ldots, N-1
$$

where, $f_{c}$ is the carrier frequency [10].

\section{SIMULATION RESULTS}

Simulation studies have been performed using convolutional coding with OFDM systems considered before. The main operating parameters of the system modeled are: carrier frequency $f_{c}=1.6 \mathrm{GHz}$, Doppler frequency $f_{D}=200 \mathrm{~Hz}$, with sub-channel carriers $N=4,16$ QAM modulation, and a block of time length $T_{b}=160 \mu \mathrm{s}$. The channel is represented by 3-path fading with one direct path and two reflected paths, with delays $\left(\mathrm{T}_{m}\right)$ equal $15 \mu \mathrm{s}$ and $30 \mu \mathrm{s}$. The parameters of the convolution coding are code rate $(r)$ equal $1 / 2$ and $1 / 3$ with constraint lengths $(K)$ equal 3 and 7 for each of them. For rate $1 / 2$ the function generators are [6,7] for constraint length 3 and $[133,171]$ for the constraint length 7 , while for rate $1 / 3$ are $[6,7,7]$ for the constraint length 3 , and $[133,145,175]$ for the constraint length 7 . All these generator vectors are represented in octal form. To validate the developed model, Fig.4 illustrates the performance of the OFDM model without coding for different values of guard interval $T_{g}$ compared with the analytical bound of 16 QAM with Doppler frequency $f_{D}=0$. It can be noted that, as expected, the performance enhanced as $T_{g}$ increases, especially with the values close to the maximum value of delay spread as shown in the two cases of $T_{g}=10 \%$ of $T_{b}$ represented by solid line with plus sign, and $20 \%$ of $T_{b}$, that exceed the value of the maximum delay spread on the channel, coincide with the analytical bound, represented by solid line with circle. While for relatively small guard intervals the degradation in performance is dominated as shown in the two cases of $T_{g}=1 \%$ of $T_{b}$ and $5 \%$ of $T_{b}$, represented by solid line with square, and solid line with star respectively.

Fig. 5 illustrates the performance obtained by convolutional coding OFDM as a function of the ratio $S / N$ at different code rates $1 / 2$ and $1 / 3$, and different constraint lengths $k=3$ and 7 for each case at $T_{g}=10 \%$ of $T_{b}$, and Doppler frequency $f_{D}=200 \mathrm{~Hz}$. It also shows the performance of the uncoded OFDM, depicted by solid line with dots. It can be noted that, at $S / N<8 \mathrm{~dB}$ for rate $1 / 2$ and at $S / N<6 \mathrm{~dB}$ for rate $1 / 3$, lower constraint length $(k=3)$, is better than higher constraint length $(k=7)$. At higher signalto-noise ratios, the performance of the convolutional code is mainly related to the free distance of the code $d_{\text {free. }}$. As the free distance of the code increases, the performance is enhanced. As shown from the figure, the performance at higher signal-to-noise ratios is better for the cases with higher free distance, which corresponds to constraint length $k=7$ for rate $1 / 3\left(d_{\text {free }}=15\right)$, and rate $1 / 2\left(d_{\text {free }}=10\right)$, represented by solid line with plus sign, and solid line with circle, compared with $k=3$ for rate $1 / 3\left(d_{\text {free }}=8\right)$, and rate $1 / 2\left(d_{\text {free }}=5\right)$, represented by solid line with star and solid line with square respectively. 
This should be taken into account when designing the system at the operating $S / N$ required.

Fig. 6 illustrates the performance of the convolutional coding OFDM at the same code rates, constraint lengths, and Doppler frequency. In this case the value of guard interval $T_{g}=20 \%$ of $T_{b}$. It can be noted that at $S / N<6.2 \mathrm{~dB}$ for rate $1 / 2$ and at $S / N<4.8 \mathrm{~dB}$ for rate $1 / 3$, lower constraint length $(k=3)$, is better than higher constraint length $(k=7)$. As shown from Fig.5 and Fig. 6 at $T_{g}=20 \%$ of $T_{b}$ the performance improvement is shifted to occur at lower $S / N$ than the case of $T_{g}=10 \%$ of $T_{b}$. One can notice that at high $S / N$ the performance of the coded OFDM in the case of rate $1 / 2$ with $k=7$ is very close to the case of rate $1 / 3$ with $k=3$.

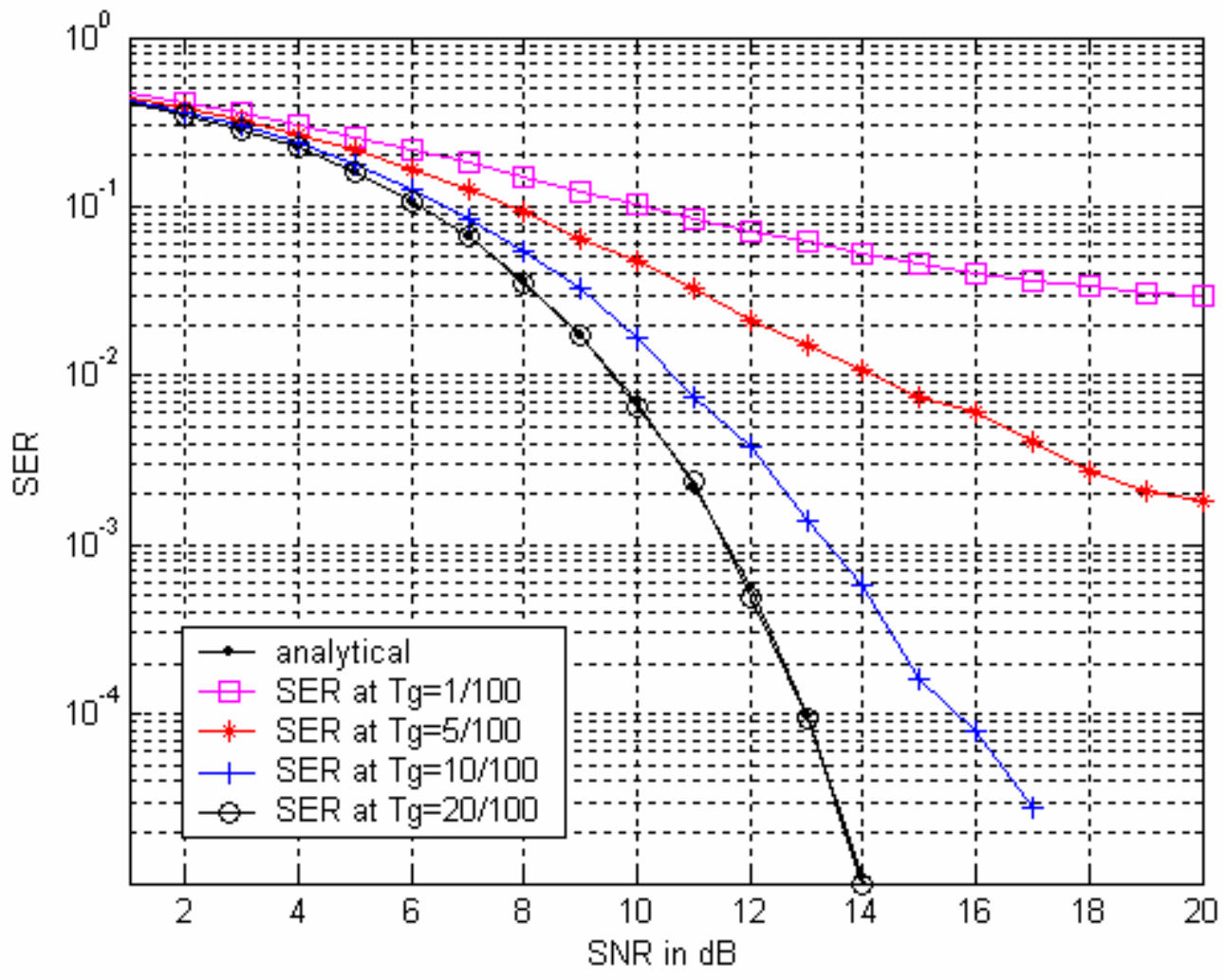

Fig. 4. Effects of $T_{g}$ on the performance of uncoded OFDM. and a comparison between simulation and theoretical bound. 


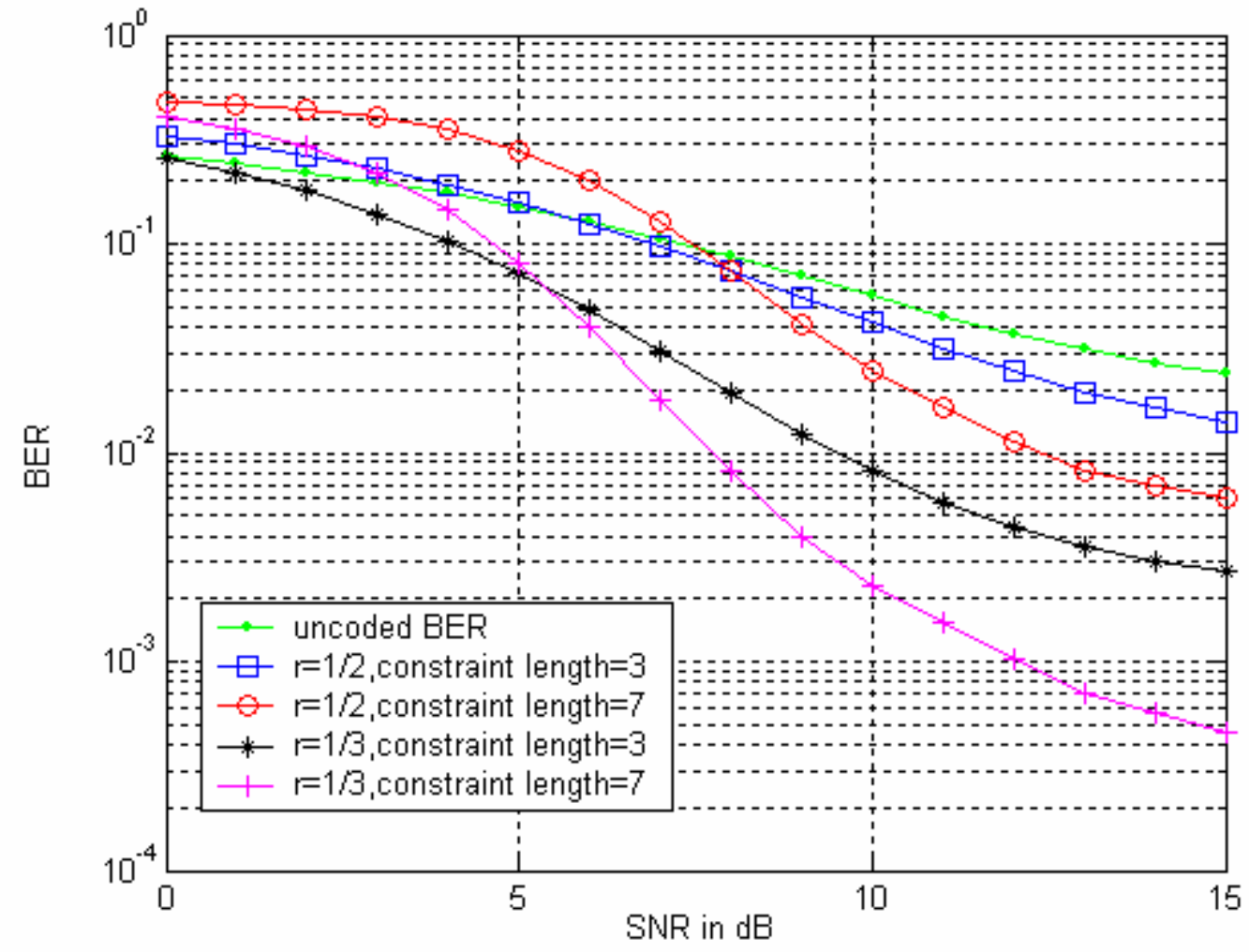

Fig.5. BER performance of the $r=1 / 2,1 / 3$ and $k=3,7$ and a comparison between uncoded and coded OFDM at $T_{g}=(10 / 100)^{*} T_{b}$.

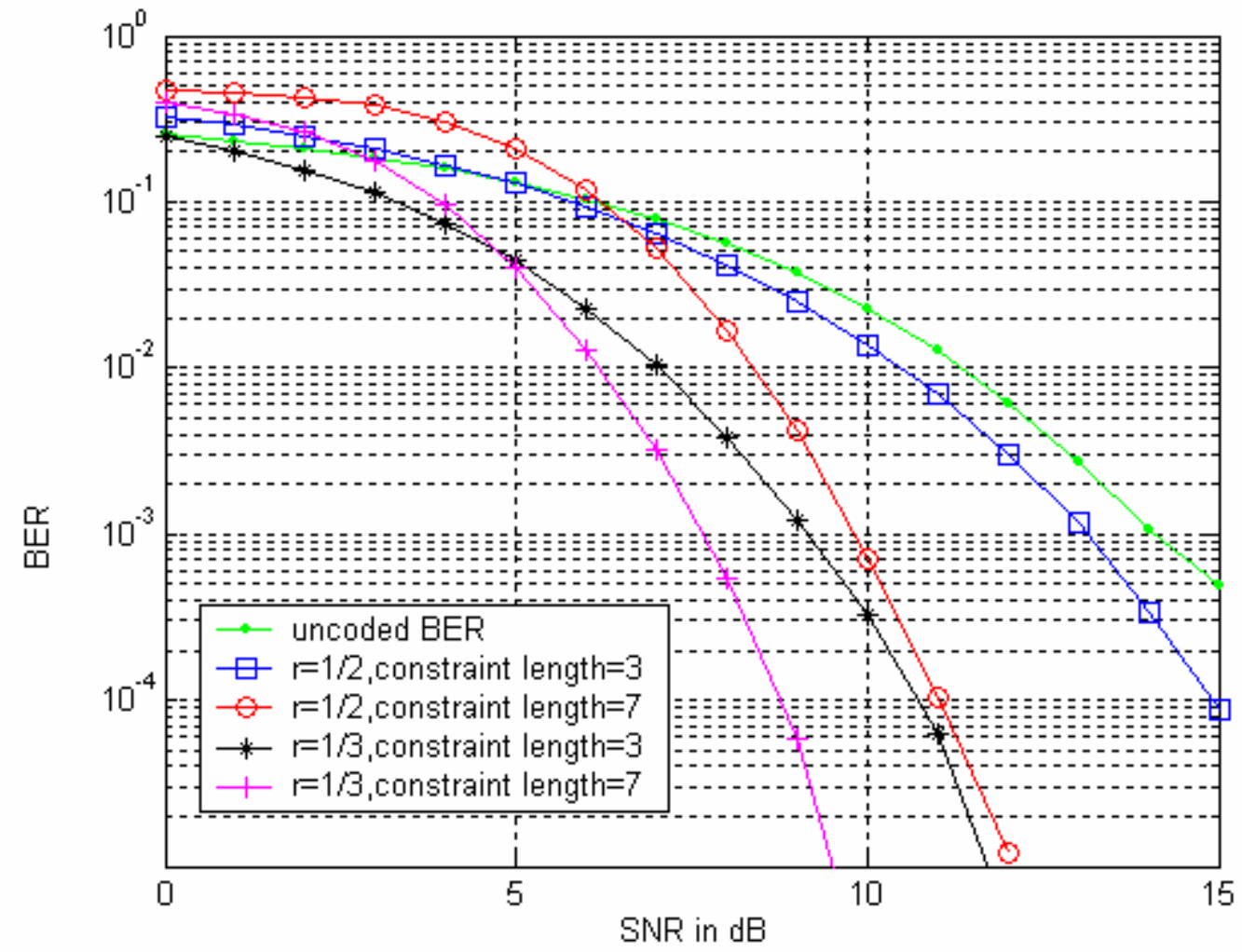

Fig.6. BER performance of the $r=1 / 2,1 / 3$ and $k=3,7$ and a comparison between uncoded and coded OFDM at $T_{g}=(20 / 100)^{\star} T_{b}$ 


\section{CONCLUSIONS}

In this paper a study of cascading of Convolutional code and orthogonal frequency division multiplexing (OFDM) is presented. Specifically studying and analyzing the effect of code rate, constraint length and time guard-interval on the bit error rate (BER) performance of a convolutional coded OFDM modulation scheme used over a multipath fading channel. Validatation of the model has been done by comparing the simulation results with the analytical bound without applying the coding stage. Discussions of the results show that, as the time guard interval, code rate, and constraint length increase the performance is improved. For different regions of signal-to-noise ratio recommendations for design issues were presented.

\section{REFERENCES}

[1] R. van Nee and R. Parsad, OFDM for wireless multimedia communications. Boston, MA: Artech House, 2000.

[2] Y. H. Kim, I. Song, H. G. Kim, T. Chang, and H. M. Kim, "Performance Analysis of a Coded OFDM System in Time-Varying Multipath Rayleigh Fading Channels," IEEE Trans. Veh. Technol., vol. 48, no.5, pp. 1610-1615, Sept. 1999.

[3] R. W. Chang, "Synthesis of Band-limited Orthogonal Signals for Multi-channel data Transmission, "Bell Syst. Tech. J., vol. 45, pp. 1775-1796, Dec. 1966.

[4] S. Darling, "On digital single-sideband modulators," IEEE Trans. Circuit Theory, vol. CT-17, pp. 409-414, Aug. 1970.

[5] P. H. Moose, "A technique for Orthogonal Frequency Division Multiplexing Frequency Offset Correction," IEEE Trans. Commun., vol 42, no. 10, pp. 29082914, Oct. 1994.

[6] Y. Mostofi, and D. C. Cox, "ICI Mitigation for Pilot-Aided OFDM Mobile Systems," IEEE Trans. Wireless Commun., vol. 4, no. 2, pp. 765-774, Mar. 2005.

[7] H. Asami and M. Sasaki, "Outline of ISDB Systems," in Proc. IEEE, vol. 94, no. 1, pp. 248-250 , Jan. 2006.

[8] S. Coleri, M. Ergen, A. Puri, and A. Bahai, "Channel Estimation Techniques Based on Pilot Arrangement in OFDM Systems," IEEE Trans. Broadcast., vol. 48, no. 3. pp.223-229, Sept. 2002.

[9] J. G. Proakis, Digital Communications. New York: McGraw-Hill, 2001.

[10] R. F. Ormondroyd and J. J. Maxey, "Comparison Of Time Guard-Band and Coding Strategies for OFDM Digital Cellular Radio in Multipath Fading," in proc. IEEE Vehicular Technology Conf., May 1997, vol. 2, pp. 850-854. 